3. These predictions are tested by examining the history of the introduction of eighteen insects to control prickly pear in Australia, and of the introduction of the ladybird to control scale insects in California. Remington's predictions are not supported.

Acknowledgments.-Miss E. Matheson supplied details of the localities for specimens of Novius cardinalis logded in the National Museum of Victoria. Professor A. M. Clark and Dr N. G. Brink read the typescript.

\title{
REFERENGES
}

ALEXANDER, M. L. 1952. Gene variability in the americana-texana-novomexicana group of Drosophila. Univ. Texas Publ. 5204, 73.

DOBZHANSKY, T., HUNTER, A.s., PAVlovsky, O., SPASSKY, B., AND WALlaCE, B. 1963. Genetics of natural populations. XXXI. Genetics of an isolated marginal population of Drosophila pseudobscurra. Genetics, 48, 91 .

DoDD, A. P. 1940. The Biological Campaign against Prickly-Pear. Commonwealth PricklyPear Board, Brisbane.

FRENCH, C. 1893. A Handbook of the Destructive Insects of Victoria. Part II; Government Printer, Melbourne.

MASON, L. G., EHRLICH, P. R., AND EMMEL, T. C. 1968. The population Biology of the butterfly, Euphydras editha. IV. Phenetics of the Jasper Ridge colony, 1965-66. Evolution, 22, 46. Elton, c. s. 1958. The Ecology of Invasions by Animals and Plants. Methuen, London.

REMrngton, C. L. 1968. The population genetics of insect introduction. Ann. Rev. Entomol., $13,415$.

TOWNSEND, J. I., JR. 1952. Genetics of marginal populations of Drosophila willistoni. Evolution, 6, 428.

TURner, J. R. G., AND williamson, M. H. 1968. Toleration of a Genetic Load. Nature, 218, 5142,700 .

\section{APOSTATIC SELECTION AND POPULATION DENSITY}

\author{
J. J. D. GREENWOOD \\ Department of Zoology, Manchester University *
}

Received 6.xii.68

R. A. FIsHer pointed out in 1930 that in a population in which rare genotypes were at a selective advantage to common ones, simply by virtue of their rarity, a polymorphism would be maintained. Later experiments made by Popham (1941, 1942) upon the predation by Rudd, Scardinius (Leuciscus) eryophthalmus L., on polymorphic populations of the aquatic bug Sigara distincta (Fieb.) showed that the fish tended to take a disproportionately high number of the commoner morphs of the bug. The work of these two authors led to the hypothesis that predators might be responsible for the maintenance of polymorphisms in populations of their prey. Cain and Sheppard (1954) and Haldane (1955) suggested that the widespread polymorphism of shellcolour and pattern in snails of the genus Cepaea (Held.) might be maintained in this way, though they did not suggest that this was likely. The evidence that predators do, indeed, behave so as to tend to maintain polymorphisms in their prey was summarised by Clarke $(1962 a, b)$, who used the term " apostatic" for the first time in this context. Clarke argued that apostatic selection had detectable effects on the polymorphism of Cepaea. However, Carter (1967) has contested Clarke's interpretation of the evidence.

* Present Address: Dept. of Biological Sciences, The University, Dundee. 
Owen $(1965 a, b)$ has very briefly considered how apostatic selection might vary in populations of different density. He studied the snail Limicolaria martensiana (Smith), in which he described four shell-pattern morphs, which varied in frequency in different populations. The dense populations seemed to be more polymorphic than the less dense ones. As a numerical index of polymorphism, I have used the probability of picking individuals of different morphs when one takes two at random from an infinite population $-\left(1-\Sigma p_{i}{ }^{2}\right)$, where $p_{i}$ is the frequency of the $i$ th morph. In a species with $M$

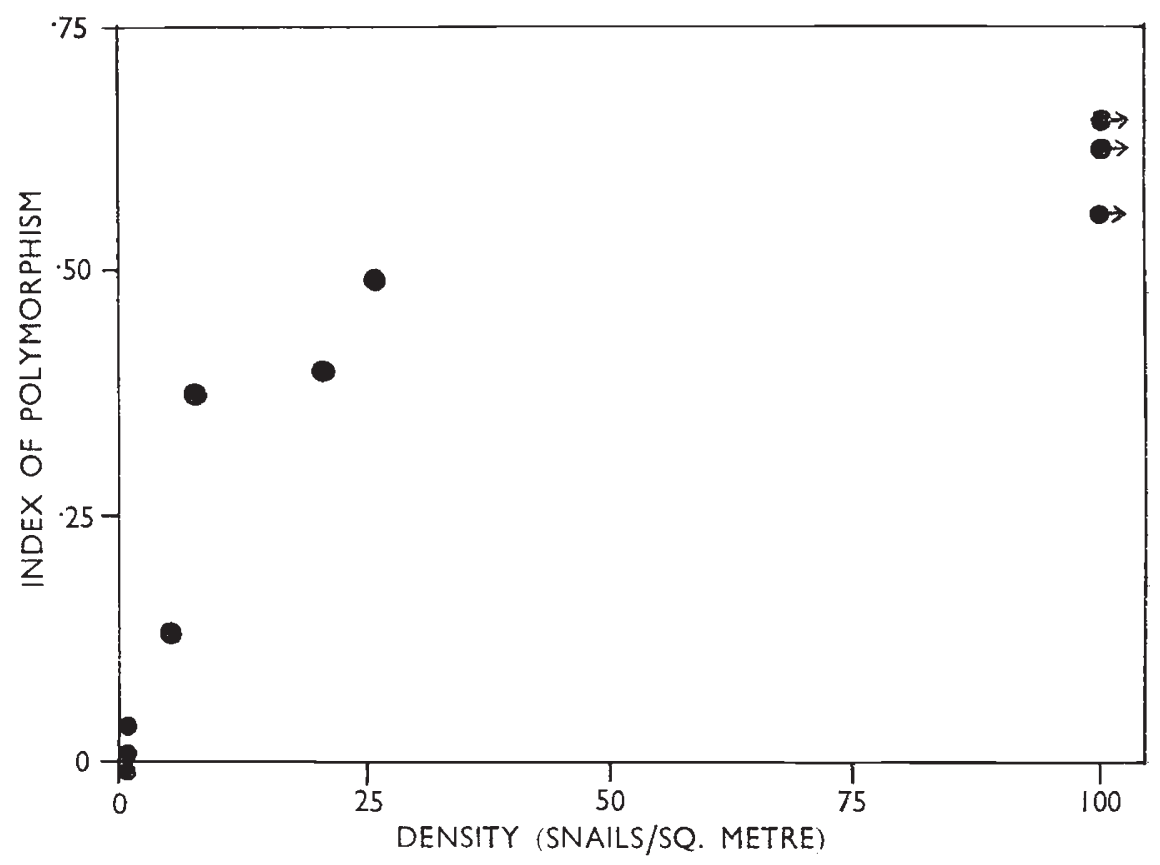

FIG. 1.-Relationship between polymorphism and population density in the snail Limicolaria martensiana. Data from Owen $(1965 a, b)$.

morphs, the maximum value of this index is $(M-1) / M$ (when the morphs are equally common), the minimum value being zero. As Simpson (1949) pointed out, for a sample from a real population an unbiased estimator of $\Sigma p_{i}{ }^{2}$ is $\left(\Sigma n_{i}\left(n_{i}-1\right)\right) /(\mathcal{N}(\mathcal{N}-1))$, where $n_{i}$ is the number of the $i$ th morph in the sample and $\mathcal{N}$ is the sample size. Using this index, I have constructed fig. 1 from Owen's data: the relation of polymorphism to population density is quite clear. Owen suggested that this relation resulted from the populations being subjected to apostatic selection and that it depended on the disproportion involved in the apostatic predation being greater at higher densities. I believe that the relationship could be produced by the action of apostatic selection even if the apostatic disproportion does not increase with density.

If, in a dimorphic population, the apparent densities of the two morphs are $D E$ and $D F$ and the numbers taken by the predator are $\mathcal{N} E$ and $\mathcal{N} F$, then the disproportion involved in the predation is $\frac{\mathcal{N E}}{\mathcal{N F}} / \frac{D E}{D F}$. This disproportion is 
greater than unity if the predator is preying apostatically. Owen assumes that the size of the disproportion increases with apparent density, $(D E+D F)$, but there is no evidence that this is so: the opposite might equally be the case. The simplest assumption is that the disproportion is independent of the density.

Due to the exquisite work of Holling (1965), there is a wealth of evidence as to the way in which the numbers of a monomorphic prey taken by a vertebrate predator in unit time change with changes in density of the prey. The relationship produces a roughly sigmoid curve (fig. 2). Two types of

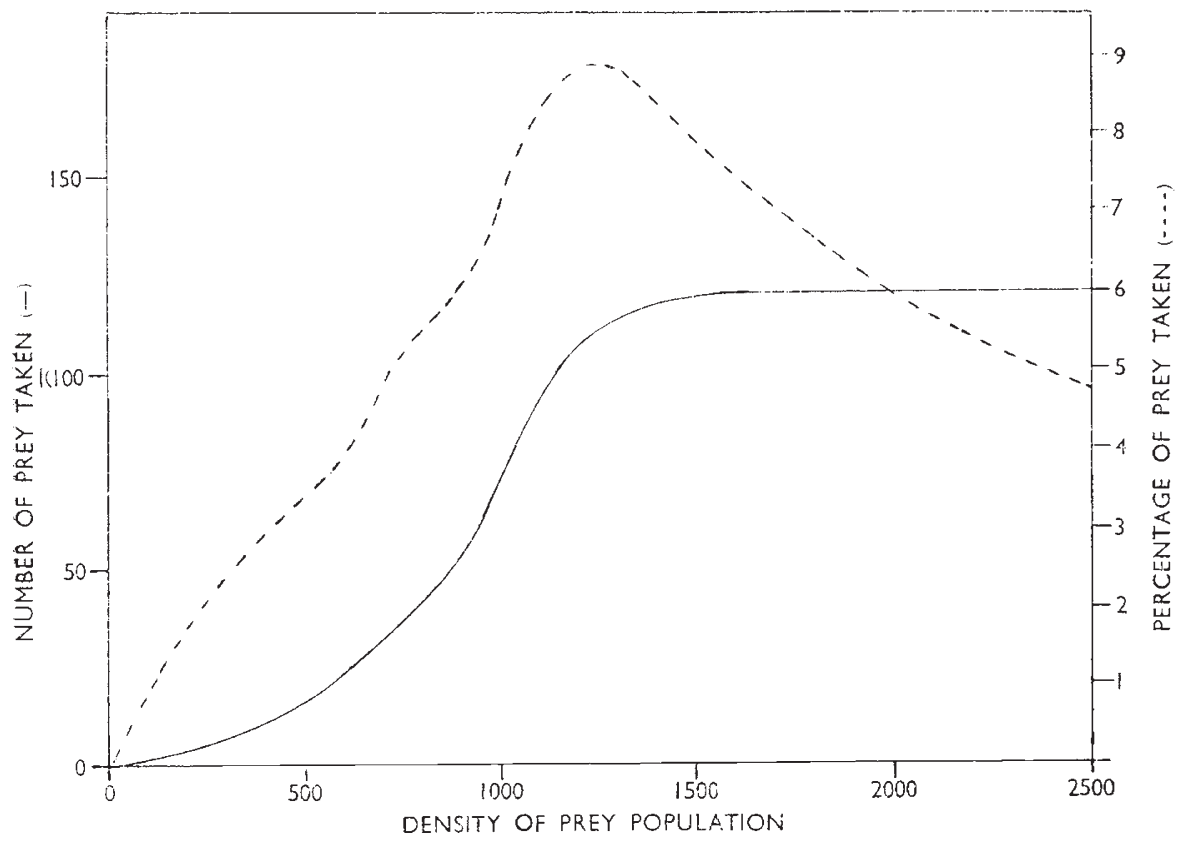

FIG. 2.-Hypothetical relationship between percentage of a prey population taken by a predator and prey density, for a given relationship between numbers of prey taken and population density.

processes cause the curve not to be a linear. Handling-time, satiation, etc. cause the slope of the curve to decrease with increasing density, so that an asymptotic value of numbers of prey taken tends to be approached at high densities. On the other hand, learning tends to accelerate the curve. Learning seems to be relatively more important at lower densities, the decelerating processes at higher densities. As a result of this relationship, in a series of populations of different densities but with the same total death rate, the proportion of deaths due to the predator will be low in populations of both low and high density and will be higher in those of intermediate density (fig. 2).

There will be few populations in which the deaths other than those caused by the apostatic predator are not selective. Let us assume, for simplicity, that the overall selection resulting from these deaths is independent of density and is such as to lead to monomorphism if it was acting alone. If, in such a situation, the apostatic disproportion is independent of density, the relative 
importance of apostatic selection will be greater at intermediate densities than at high or low densities.

In some situations the relative importance of apostatic selection will be so small at all densities that it is always outweighed by the frequency independent selection: populations of all densities will be monomorphic. In all other cases, populations at low densities and at high densities will be monomorphic, since at such densities the relative importance of frequency independent selection is great enough to outweigh apostatic selection. There will, however, be an intermediate range of densities in which apostatic selection is of great enough relative strength to lift the population away from the monomorphism towards which the frequency independent selection is tending to drive it. The level of polymorphism reached at any particular density will depend on how much more important than the frequency independent selection is the apostatic selection.

If one considers a polymorphic, rather than a dimorphic popultion, it seems likely that the situation will be similar. Consider four morphs, $A, B$, $C$, and $D$, of which $A$ is most favoured by the frequency independent selection and $D$ the least favoured. There will be a range of densities over which $D$ will be maintained in the population with the other morphs. It will lie totally within the somewhat wider range over which $C$ is maintained, which itself will lie within the even wider range over which $B$ is maintained. Outside this range apostatic selection is relatively so unimportant that not even $B$ can be maintained and the population is monomorphic for $A$. If one were to investigate a series of populations of increasing density, their contained morphs would be $A ; A, B ; A, B, C ; A, B, C, D ; A, B, C ; A, B ; A$. It may well be that Owen's populations of Limicolaria martensiana lie in the first part of such a range of densities.

If the apostatic disproportion, $\frac{\mathcal{N E}}{\mathcal{N F}} / \frac{D E}{D F}$, tends to be higher at higher densities the relation of polymorphism to density will be broadly similar to that obtaining when the disproportion is independent of density. It will differ in that the rise of index of polymorphism with increasing density will be steeper and will continue at rather higher densities. Since the relative importance of apostatic selection will not fall until higher population densities are reached, the polymorphism will be maintained over a longer range. It is, indeed, possible to imagine a situation in which the increase in the disproportion with increasing density continued to outstrip the decline in proportion of the deaths caused by the apostatic predator. In such a case, apostatic predation would never be overcome, even in populations of the highest density; the range of densities over which the population was polymorphic would extend upwards to infinity.

If the apostatic disproportion was to decrease with increasing population density, the range of densities over which apostatic selection overcame frequency independent selection would be reduced, compared with the situation in which the disproportion was constant. If the decrease was sufficiently rapid, indeed, the initial rise in proportion of deaths due to predation might be insufficient to counterbalance it: there would be no densities at which the magnitude of apostatic selection was sufficiently great to maintain a polymorphism.

Thus, if apostatic selection is responsible for maintaining a polymorphism in a series of populations, one would expect that the relationship of index 
of polymorphism to population density would be positive at lower densities and negative at higher densities: the populations of lowest and highest densities would be monomorphic. Until one knows how the magnitude of the apostatic disproportion is related to the density of the prey and to the relative frequencies of the morphs of the prey, it will be impossible to predict the form of the relationship more precisely.

\section{Summary}

1. It is shown that if apostatic selection is responsible for maintaining polymorphism in a species then the relation of degree of polymorphism to density will be positive at first, then negative. Both low and high density populations will be monomorphic, intermediate density populations being polymorphic.

2. The general form of the relation is almost independent of the form of the relation between the size of the apostatic differential and the population density.

Acknowledgments.-I wish to thank Professor A. J. Cain, my supervisor, and Dr L. M. Cook for advice, encouragement and stimulation. Thanks are also due to Professors Cain and $R$. Dennell for the facilities with which $I$ have been provided in the University of Manchester Zoology Department.

During this work I was supported by the Phillip Buckle Research Scholarship in Agricultural Zoology in the University of Manchester (2 years) and a Science Research Council Studentship (1 year).

\section{REFERENCES}

GAIN, A. J., AND SHEPPARD, P. M. 1954. Natural selection in Cepaea. Genetics, 39, 89-116. GARTER, M. A. 1967. Selection in mixed colonies of Cepaea nemoralis and Cepaea hortensis. Heredity, 22, 117-139.

CLARKE, B. G. 1962a. Balanced polymorphism and the diversity of sympatric species. In Taxonomy and Geography, ed. D. Nicholls, Syst. Ass. Publ. No. 4.

GLARKE, B. c. 1962 b. Natural selection in mixed populations of two polymorphic snails. Heredity, 17, 319-345.

FIsHer, R. A. 1930. The Genetical Theory of Natural Selection. Clarendon, Oxford.

HALDANE, J. B. S. 1955. On the biochemistry of heterosis, and the stabilization of polymorphism. Proc. roy. Soc. B., 144, 217-220.

Holling, c. s. 1965. The functional response of predators to prey density and its role in mimicry and population regulation. Mem. Ent. Soc. Can., 45, 1-60.

POPHAM, E. J. 1941. The variation in the colour of certain species of Arctocorisa (Hemiptera: Corixidae) and its significance. Proc. Zool. Soc. Lond. A., 111, 135-172.

POPHAM, E. J. 1942. Further experimental studies on the selective action of predators.

Proc. Zool. Soc. Lond. A., 112, 105-117.

OWEN, D. F. 1965a. Density effects in polymorphic land snails. Heredity, 20, 312-315.

OWEN, D. F. 1965b. A population study of an equatorial land snail, Limicolaria martensiana (Achatinidae). Proc. Zool. Soc. Lond., 144, 361-381.

simpson, E. H. 1949. Measurement of diversity. Nature, 163, 668. 